\title{
THE RELATIONSHIP BETWEEN THE ZEROS OF BEST APPROXIMATIONS AND DIFFERENTIABILITY
}

\author{
PETER B. BORWEIN ${ }^{1}$
}

\begin{abstract}
We examine the relationship between the analytic properties of continuous functions on $[-1,1]$ and the location of the roots of the sequence of best polynomial approximations. We show that if the approximants have no zeros in a certain ellipse then the function being approximated must be analytic in this ellipse. We also show that the rate at which the zeros of the $n$th approximant tend to the interval $[-1,1]$ determines the global differentiability of the function under consideration.
\end{abstract}

1. Introduction. The theorems of Bernstein and Jackson establish an exact relationship between the rate of convergence of the sequence of best polynomial approximants to a function $f \in C[-1,1]$ and the global differentiability of $f$ on $[-1,1]$ (see $[\mathbf{1}$ or $\mathbf{3}]$ ). We intend to show that such a relationship also holds between the location of the zeros of the sequence of best approximants and the global differentiability of $f$.

Let $C[-1,1]$ be the set of continuous functions on $[-1,1]$. Let $\pi_{n}$ denote the collection of algebraic polynomials of degree at most $n$ with real coefficients. For $f \in C[-1,1]$ let

$$
P_{n}^{*}(f):=\min _{p_{n} \in \pi_{n}}\left\|f-p_{n}\right\|_{[-1,1]}
$$

where $\|\cdot\|_{[a, b]}$ denotes the supremum norm on $[a, b]$. Let $P_{n}(f)$ be the best uniform approximation to $f$ from $\pi_{n}$. Then $P_{n}(f)$ is, of course, the unique polynomial of degree at most $n$ which attains the minimum in (1).

Let $E_{\rho}, \rho \geq 1$, be the open ellipse (in the complex plane $\mathbf{C}$ ) with foci at \pm 1 and with axes $\frac{1}{2}\left(\rho \pm \rho^{-1}\right)$.

We will prove the following theorems:

THEOREM 1. Let $N$ be an integer. Suppose $f \in C[-1,1]$ and, for all $n \geq N$, $P_{n}(f)$ has no zeros in $E_{\rho}$. Then $f$ is analytic in $E_{\rho}$ (that is, $f$ is the restriction to $[-1,1]$ of a function analytic in $\left.E_{\rho}\right)$.

THEOREM 2. Let $N$ be an integer. Suppose $f \in C[-1,1]$ and there exist integers $k$ and $N$ and $a \delta \in(0,1)$ so that, for each $n \geq N, P_{n}(f)$ has no zeros in $E \rho_{n}$, where $\rho_{n}=n^{(1+k+\delta) / n}$. Then, for any $\varepsilon>0, f$ is $k$ times continuously differentiable on $[-1+\varepsilon, 1-\varepsilon]$ and $f^{(k)}$ satisfies a Lipschitz condition of order $\delta$ on $[-1+\varepsilon, 1-\varepsilon]$.

Theorem 1 is an analogue of the observation that if a formal power series $\sum_{n=0}^{\infty} a_{n} z^{n}$ has the property that, for each $N, \sum_{n=0}^{N} a_{n} z^{n}$ has no zeros in

Received by the editors November 15, 1982.

1980 Mathematics Subject Classification. Primary 41A50.

${ }^{1}$ Research supported in part by N.S.E.R.C. of Canada. 
$C_{\rho}=\{|z|<\rho\}$, then $\sum_{n=0}^{\infty} a_{n} z^{n}$ is convergent in $C_{\rho}$. This is straightforward. We can write, for $a_{N} \neq 0$,

$$
\sum_{n=0}^{N} a_{n} z^{n}=a_{N} \prod_{i=1}^{N}\left(z-z_{i}\right), \quad\left|z_{i}\right|>\rho,
$$

and thus,

$$
\left|a_{N}\right| \prod_{i=1}^{N}\left|z_{i}\right|=\left|a_{0}\right|
$$

In particular,

$$
\left|a_{N}\right|^{1 / N} \leq\left|a_{0}\right|^{1 / N} / \rho
$$

from whence the observation follows.

Jentzsch's Theorem says that the zeros of the partial sums of $f(z)=\sum_{n=0}^{\infty} a_{n} z^{n}$ are dense in $\{|z|=\rho$, where $\rho$ is the radius of convergence of $f$. Walsh [5] offers the following analogue of Jentzsch's Theorem.

THEOREM 3. If $E_{\rho}$ is the maximal ellipse of analyticity for $f$, then the zeros of $P_{n}(f)$ are dense in the boundary of $E_{\rho}$.

If we had assumed, in Theorem 1 , that $f$ was analytic on $[-1,1]$, instead of just continuous, then we could have deduced the result from this analogue of Jentzsch's Theorem. Professor Gončar, in private communication, informs me that he can prove the following interesting generalization: If there exists a domain $D, D \cap$ $[-1,1] \neq \varnothing$, so that no $P_{n}(f)$ has any zero in $D$, then $f$ is analytic in $D$. This generalization and other extensions of Theorem 1 to general compact sets were discussed by H. P. Blatt and E. B. Saff at the Tampa Conference on Rational Approximation and Interpolation in December 1983. Related problems for Padé approximants are considered by Gončar in [2].

2. An inequality for polynomials with no roots in $E_{\rho}$. The proofs of Theorems 1 and 2 are consequences of the following inequality: Then

INEQUALITY 1. Suppose $p_{n}(x)=\sum_{k=0}^{n} a_{k} x^{k} \in \pi_{n}$ and $p_{n}$ has no zeros in $E_{\rho}$.

$$
\left|a_{n}\right| \leq\left(2^{n} / \rho^{n}\right)\left\|p_{n}\right\|_{[-1,1]}
$$

PROOF. Let

$$
q_{2 n}(z)=z^{n} p_{n}\left(\left(z+z^{-1}\right) / 2\right) .
$$

We note that $q_{2 n} \in \pi_{2 n}$ has lead coefficient $a_{n} / 2^{n}$ and that if $z_{0}$ is a root of $q_{2 n}$ then so are $1 / z_{0}$ and $\bar{z}_{0}$. Since $w=\left(z+z^{-1}\right) / 2$ maps $\mathbf{C}-\{z|1 / \rho<| z \mid<\rho\}$ into $\mathrm{C}-E_{\rho}$, it follows that

$$
q_{2 n}(z)=\frac{a_{n}}{2^{n}} \prod_{i=1}^{n}\left(z-\alpha_{i}\right) \prod_{i=1}^{n}\left(z-\frac{1}{\alpha_{i}}\right)
$$


where $\left|\alpha_{i}\right| \geq \rho$ for each $i$ and where the $\alpha_{i}$ come in conjugate pairs. Thus, for $|z|=1$,

$$
\begin{aligned}
\left|q_{2 n}(z)\right| & =\left|\frac{z^{n} a_{n}}{2^{n}} \prod_{i=1}^{n}\left(z-\alpha_{i}\right) \prod_{i=1}^{n} \frac{\left(\alpha_{i}-1 / z\right)}{\alpha_{i}}\right| \\
& =\frac{\left|a_{n}\right|}{2^{n}} \frac{\left|\prod_{i=1}^{n}\left(z-\alpha_{i}\right) \prod_{i=1}^{n}\left(\bar{z}-\alpha_{i}\right)\right|}{\left|\prod_{i=1}^{n} \alpha_{i}\right|} \\
& =\frac{\left|a_{n}\right|}{2^{n}} \frac{\left|\prod_{i=1}^{n}\left(z-\alpha_{i}\right)\right|^{2}}{\left|\prod_{i=1}^{n} \alpha_{i}\right|}
\end{aligned}
$$

By the maximum modulus principle

$$
\left\|\prod_{i=1}^{n}\left(z-\alpha_{i}\right)\right\|_{\{|z|=1\}} \geq\left|\prod_{i=1}^{n} \alpha_{i}\right|
$$

and, hence,

$$
\left\|p_{n}\right\|_{[-1,1]}=\left\|q_{2 n}\right\|_{\{|z|=1\}} \geq \frac{\left|a_{n}\right|}{2^{n}}\left|\prod_{i=1}^{n} \alpha_{i}\right| \geq\left|a_{n}\right| \frac{\rho^{n}}{2^{n}}
$$

There exists $p_{n} \in \pi_{n}, p_{n}=2^{n} x^{n}+\cdots$, so that, in the variable $x=\left(z+z^{-1}\right) / 2$,

$$
p_{n}\left(\frac{z+z^{-1}}{2}\right)=\left(z^{n}-\rho^{n}\right)\left(z^{-n}-\rho^{n}\right) /\left(-\rho^{n}\right) .
$$

For this polynomial $a_{n}=2^{n}$ and

$$
\left\|p_{n}\right\|_{[-1,1]} \leq\left\|z^{n}-\rho^{n}\right\|_{\{|z|=1\}}^{2} / \rho^{n} \leq \rho^{n}+2+\rho^{-n}
$$

or

$$
\left|a_{n}\right| \geq\left(2^{n} /\left(\rho^{n}+2+\rho^{-n}\right)\right)\left\|p_{n}\right\|_{[-1,1]} .
$$

In particular, Inequality 1 is asymptotically best possible.

Minor modifications to the proof of Inequality 1 yield:

INEQUALITY 2. Suppose $p_{n}(x)=\sum_{k=0}^{n} a_{n} x^{k} \in \pi_{n}$ and $p_{n}$ has $k$ or fewer zeros in $E_{\rho}$. Then

$$
\left|a_{n}\right| \leq\left(2^{n} / \rho^{n-k}\right)\left\|p_{n}\right\|_{[-1,1]} .
$$

ProOF OF ThEOREMS 1 AND 2 . Let $n \geq N$ and let $a_{n}$ be the lead coefficient of $P_{n}(f)$. From Inequality 1 and the assumptions on the roots of $p_{n}$ we have

$$
\left|a_{n}\right| \leq\left(2^{n} / \rho^{n}\right)\left\|P_{n}(f)\right\|_{[-1,1]} \leq\left(2^{n} / \rho^{n}\right) M,
$$

where $M=2\|f\|_{[-1,1]}$. It follows that there exists $S_{n-1} \in \pi_{n-1}$ so that

$$
\left\|S_{n-1}-P_{n}(f)\right\|_{[-1,1]} \leq 2 M / \rho^{n} \text {. }
$$

One need only set $P_{n}(f)-S_{n-1}=a_{n} T_{n} / 2^{n-1}$, where $T_{n}$ is the $n$th Cebyčev polynomial (see $[3$, p. 31]). Thus,

$$
\begin{aligned}
P_{n}^{*}(f) & \leq\left\|f-S_{n}\right\|_{[-1,1]} \leq\left\|f-P_{n+1}\right\|_{[-1,1]}+\left\|S_{n}-P_{n+1}\right\|_{[-1,1]} \\
& \leq P_{n+1}^{*}(f)+2 M / \rho^{n+1}
\end{aligned}
$$


and

$$
P_{n}^{*}(f) \leq 2 M \sum_{m=1}^{\infty} \frac{1}{\rho^{n+m}} \leq \frac{C}{\rho^{n}},
$$

where $C$ is independent of $n$. This, by Bernstein's characterization of analytic functions in terms of the rate of convergence of $P_{n}^{*}$, guarantees that $f$ is analytic in $E_{\rho}$. (See, for example, $[3$, p. 76].)

The proof of Theorem 2 proceeds along similar lines. Equations (2)-(5) become

$$
\begin{gathered}
\left|a_{n}\right| \leq 2^{n} M / n^{1+k+\delta}, \\
\left\|S_{n-1}-P_{n}(f)\right\|_{[-1,1]} \leq 2 M / n^{1+k+\delta}, \\
P_{n}^{*}(f) \leq P_{n+1}^{*}(f)+2 M / n^{1+k+\delta},
\end{gathered}
$$

and

$$
P_{n}^{*}(f) \leq C^{\prime} / n^{k+\delta} .
$$

Equation $\left(5^{\prime}\right)$, once again by Bernstein's results $[3$, p. 61$]$, guarantees that $f$ has $k$ continuous derivatives on $[-1+\varepsilon, 1-\varepsilon]$ and $f^{(k)} \in \operatorname{Lip}_{\delta}$ on $[-1+\varepsilon, 1-\varepsilon]$.

If we use Inequality 2 instead of Inequality 1 in the above proofs, we can deduce that both Theorems 1 and 2 hold under weaker assumptions. For example, we need only assume that the number of zeros of $P_{n}(f)$ in $E_{\rho}$ (or $\left.E_{\rho_{n}}\right)$ is $o(n)$.

3. How sharp are Theorems 1 and 2? If $f$ is analytic and nonzero in $E_{\rho}$ then the sequence $\left\{P_{n}\right\}$ converges uniformly to $f$ on compact subsets of $E_{\rho}[\mathbf{3}, \mathrm{p}$. 76]. Hence, for $\rho^{\prime}<\rho$ and for $n$ sufficiently large, the zeros of $P_{n}$ will lie outside $E_{\rho^{\prime}}$. In particular, Theorem 1 can be used to characterize the largest ellipse in which $f$ is analytic.

For nonanalytic $f$ we have the following example: Let $k$ be a positive integer and let

$$
f(x):=5+\sum_{m=1}^{\infty}\left(3^{-m}\right)^{k} T_{3^{m}}(x),
$$

where $T_{i}$ is the $i$ th Čebyčev polynomial on $[-1,1]$. Then, for $3^{h} \leq n<3^{h+1}$,

$$
P_{n}(f)=5+\sum_{m=1}^{h}\left(3^{-m}\right)^{k} T_{3^{m}}(x) .
$$

(See $[\mathbf{1}$, p. 132].) Also,

$$
\left\|T_{m}\right\|_{E_{\rho}} \leq \rho^{m}
$$

(See $\left[3\right.$, p. 42].) For $h$ sufficiently large, if $\rho<3^{k h / 3^{h}}$ then

$$
\left\|\sum_{m=1}^{h}\left(3^{-m}\right)^{k} T_{3^{m}}\right\|_{E_{\rho}} \leq \sum_{m=1}^{h}\left(\frac{3^{3^{(m-h)} h}}{3^{m}}\right)^{k}<5 .
$$

Thus, for $n$ sufficiently large, $P_{n}(f)$ has no zeros in $E_{\rho}$, where $\rho=n^{k / n}$. However, by examining $f(\cos \theta)$ one can verify that $f$ is not $k$ times continuously differentiable on $[-1+\varepsilon, 1-\varepsilon]$. 
The previous example leaves a gap of $1+\delta$ between the assumptions on $k$ in Theorem 2 and the "best possible" assumptions.

The results of this paper are quintessentially results about best approximants. Given $f \in C[-1,1]$ and any compact sets $K$ in the complex plane that separates $[-1,1]$ from infinity, it is always possible to find a sequence of polynomials with all roots in $K$ that converges to $f$. (See, for example, $[\mathbf{4}]$.)

\section{REFERENCES}

1. E. W. Chency, Introduction to approximation theory, McGraw-Hill, New York, 1966.

2. A. A. Gončar, On uniform convergence of Padé approximants, Math. USSR Sb. 44 (1983), 539 559.

3. G. G. Lorentz, Approximation of functions, Holt, Rinehart and Winston, New York, 1966.

4. M. D. Thompson, Approximation by polymomials whose zeros lie on a curve, Duke Math. J. 31 (1964), 255-265.

5. J. L. Walsh, The analogue for maximally convergent polymomials of Jentzsch's theorem, Duke Math. J. 26 (1959), 605-616.

Department of Mathematics, Statistics and Computing SCience. Dalhousie University, Halifax, Nova Scotia, Canada 